\title{
ANÁLISIS FITOLIITICOS EN SUELOS FORMADOS SOBRE ANTIGUAS TERRAZAS DEL RIOO URUGUAY (COLÓN, ENTRE RÍOS): PRIMERA EVIDENCIA DE RETRACCIÓN DE LOS PALMARES DE BUTIA YATAY (MART.) BECC.
}

\author{
NOELIA ISABEL PATTERER ${ }^{1}$ \\ ALEJANDRO FABIÁN ZUCOL \\ ESTEBAN PASSEGGI ${ }^{1}$
}

\footnotetext{
1'Laboratorio de Paleobotánica, Centro de Investigaciones Científicas y Transferencia de Tecnología a la Producción (CICyTTP-CONICET), Dr. Materi y España SN, E3105BWA, Diamante, Entre Ríos, Argentina.
}

Recibido: 16 de agosto de 2016 - Aceptado: 20 de marzo de 2017

Para citar este artículo: Noelia Isabel Patterer, Alejandro Fabián Zucol y Esteban Passeggi (2017). Análisis fitolíticos en suelos formados sobre antiguas terrazas del Río Uruguay (Colón, Entre Ríos): primera evidencia de retracción de los palmares de Butia yatay (Mart.) Becc. Publicación Electrónica de la Asociación Paleontológica Argentina 17 (1): 1-13.

Link a este artículo: http://dx.doi.org/10.5710/PEAPA.20.03.2017.122

DESPLAZARSE HACIA ABAJO PARA ACCEDER AL ARTÍCULO

Asociación Paleontológica Argentina Maipú $6451^{\circ}$ piso, C1006ACG, Buenos Aires República Argentina Tel/Fax (54-11) 4326-7563 Web: www.apaleontologica.org.ar

Otros artículos en Publicación Electrónica de la APA 16(2):

\section{Deschamps \& Tomassini}

LATE CENOZOIC VERTEBRATES FROM THE SOUTHERN PAMPEAN REGION: SYSTEMATIC AND BIO-CHRONOSTRATIGRAPHIC UPDATE

\section{Borromei et al.}

POSTGLACIAL VEGETATION AND CLIMATE CHANGES INFERRED FROM A PEAT POLLEN RECORD IN THE RÍO PIPO VALLEY, SOUTHERN TIERRA DEL FUEGO 


\title{
ANÁLISIS FITOLÍTICOS EN SUELOS FORMADOS SOBRE ANTIGUAS TERRAZAS DEL RÍO URUGUAY (COLÓN, ENTRE RÍOS): PRIMERA EVIDENCIA DE RETRACCIÓN DE LOS PALMARES DE BUTIA YATAY (MART.) BECC.
}

\author{
NOELIA ISABEL PATTERER ${ }^{1}$, ALEJANDRO FABIÁN ZUCOL ${ }^{1}$ Y ESTEBAN PASSEGGI ${ }^{1}$
}

${ }^{1}$ Laboratorio de Paleobotánica, Centro de Investigaciones Científicas y Transferencia de Tecnología a la Producción (CICyTTP-CONICET), Dr. Materi y España SN, E3105BWA, Diamante, Entre Ríos, Argentina.noepatt1@hotmail.com; cidzucol@gmail.com; estebanpasseggi@cicyttp.org.ar

\begin{abstract}
Resumen. Los palmares de Butia yatay (Mart.) Becc. en el Parque Nacional El Palmar (Colón, Entre Ríos), son comunidades relictuales que se desarrollan sobre los suelos arenosos que pertenecen al orden de los Entisoles. Estos suelos constituyen las terrazas antiguas del río Uruguay. La serie de suelos Puerto Yeruá son suelos arenosos pardos formados sobre terrazas y poseen un mayor desarrollo pedológico. Se ha estudiado la composición fitolítica de la secuencia pedológica de esta serie de suelos con la finalidad de contribuir al conocimiento de la flora que acompañó el desarrollo de los mismos. Las asociaciones fitolíticas observadas reflejan el cambio de dos ciclos pedológicos, que dividen al perfil analizado en dos zonas demarcadas por la presencia de diferentes paleocomunidades: una de palmares asociados a un estrato herbáceo pobre y una superior de pastizales meso-megatérmicos con escasa presencia de microrestos afines a elementos arbóreos. El registro fitolítico descripto en el presente trabajo es el primer antecedente que muestra el posible punto de cambio en la fisonomía del paisaje y la retracción de los palmares de yatay en el área de estudio.
\end{abstract}

Palabras clave. Silicofitolitos. Serie Puerto Yeruá. Parque Nacional El Palmar. Río Uruguay. Paleoambientes.

\begin{abstract}
PHYTOLITH ANALYSIS OF SOILS FORMED OVER OLD TERRACES OF THE URUGUAY RIVER (COLÓN, ENTRE RÍOS). FIRST EVIDENCE OF BUTIA YATAY (MART.) BECC. PALM FOREST RETRACTION. The Butia yatay (Mart.) Becc. palm forest in the El Palmar National Park (Colón, Entre Ríos) is part of a relict community developed over sandy soil, belonging to the Entisols order of the old terraces of the Uruguay River. The Puerto Yeruá soil series are gray sandy soils formed on terraces that have greater pedological development. The phytolith assemblages of this pedological sequence have been studied in order to contribute to the knowledge of the flora that accompanied the development of these series. The phytolith associations observed has reflected the change of two pedological cycles in which the analyzed profile is divided, with two zones demarcated by the presence of different paleocommunities: one is a palm forest associated with a poor herbaceous stratum, and an upper stratum of meso-megathermic grassland with scarce presence of remains with affinity to arboreal elements. The phytolith record described in this paper is the first record that shows the possible location of the change in the physiognomy of the landscape and the retraction of the Butia yatay palms in the study area.
\end{abstract}

Key words. Silicophytoliths. Puerto Yeruá soil series. El Palmar National Park. Uruguay River. Paleoenvironments.

DURANTE la década del '30, Báez (1937) realiza el primer análisis sobre la distribución de las palmeras de Butia yatay (Mart.) Becc. en la provincia de Entre Ríos, definiendo cuatro núcleos de yatay que serían los puntos más australes de la distribución de esta especie en Argentina: uno en la isla El Palmar (río Paraná), un segundo -con pocos ejemplares- en el Distrito Palmar, Departamento Diamante, un tercero en Campo Racedo, Departamento Villaguay, y el cuarto en el Palmar Grande, Departamento Colón. Luego, el propio Báez
(1942) propone una amplia distribución de B. yatay para el territorio entrerriano, sobre todo en la costa del río Uruguay, en asociación con suelos principalmente arenosos. Entre los años 1948 y 1949, Crovetto y Piccinini (1951) realizaron un inventario fitosociológico de los palmares de $B$. yatay en las provincias de Entre Ríos, Santa Fe y Corrientes, y describieron los palmares más importantes de Entre Ríos. En la actualidad, aquellos palmares distribuidos en mayor o menor medida en las provincias mesopotámicas de Argentina 
quedan resguardados bajo la figura de Parque Nacional: 1) El Palmar (PNEP) creado en 1965 y localizado en el centroeste de la provincia de Entre Ríos, sobre el río Uruguay en el Departamento Colón y 2) el Parque Nacional Mburucuyá, creado en el año 2001 y ubicado en la provincia de Corrientes, en un área protegida de 17660 ha. Numerosos han sido los factores que han determinado el estado actual de los núcleos de yatay en Argentina, principalmente las actividades agropecuarias, forestales y citrícolas han reemplazado grandes extensiones de palmares por cultivos anuales o de cítricos y forestaciones. Un ejemplo puede ser observado claramente en el núcleo de Campo Racedo (Departamento Villaguay, Entre Ríos) en donde los ejemplares de yatay se encuentran en campos cultivados. Los remanentes de palmares que no han sido afectados por estas actividades sufrieron desde hace varios siglos una fuerte presión de pastoreo y el recurrente uso del fuego para el manejo ganadero. Una de las características comunes de los núcleos de yatay remanentes es la homogeneidad etaria de los individuos; la edad del Palmar Grande de Colón ha sido estimada en unos 250 años (Lunazzi, 2009). En síntesis, la conservación general de este sistema se ve afectado por diversos problemas como la invasión de especies arbóreas exóticas y el rol de los incendios estacionales en la dinámica de esta vegetación (Menvielle, 2003 en Goveto, 2005), como así también el control del fuego en áreas protegidas y la expansión cada vez mayor de las poblaciones de árboles nativos en el paisaje (Rolhauser, 2002).

Geomorfológicamente, los palmares de yatay en la costa del río Uruguay están asociados directamente a suelos originados en los afloramientos de la Formación El Palmar (Pleistoceno superior), la que se distribuye en una faja de 4 a $15 \mathrm{~km}$ de ancho a lo largo de la margen derecha del río Uruguay, aflorando en el este de la provincia de Corrientes y Entre Ríos hasta Concepción del Uruguay (Iriondo y Kröhling, 2008). Pedogenéticamente los suelos arenosos rojos del río Uruguay pueden considerarse como suelosrelictos o Paleosoles. Estos suelos se agrupan en suelos arenosos rojizos (Serie Yuquerí Chico y Serie Yuquerí Grande) y suelos arenosos pardos (Serie Puerto Yeruá y Serie Mandisovī) (sensu Tasi, 2009).

Entre los vegetales productores de fitolitos, y en particular de silicofitolitos, las gramíneas y las ciperáceas son las familias de mayor importancia en lo que respecta a las características cuali-cuantitativas de producción (Piperno, 1998; Pearsall, 2000). No obstante, existen grupos vegetales cuya producción fitolítica reviste importancia por el elevado poder diagnóstico de sus morfotipos. Las palmeras se caracterizan por la presencia de fitolitos denominados estegmatas (Tomlinson, 1961). Los tipos morfológicos más frecuentes son esféricos a elipsoidales espinosos y cónicos. Recientemente, los análisis fitolíticos en diferentes formaciones geológicas de la región avalan la presencia de este tipo de comunidad vegetal en el pasado y han demostrado ser muy abundantes y diversas. Entre ellas, para el Mioceno superior la Formación Paraná (Zucol y Brea, 2000a,b), para el Pleistoceno inferior la Formación Puerto General Alvear (Zucol y Brea, 2001), para el Pleistoceno superior-Holoceno inferior la Formación Tezanos Pinto (Erra, 2010; Erra et al., 2011, 2013) en la Cuenca del río Paraná y para el Plio-Pleistoceno inferior Formación San Salvador (Patterer, 2015) y para el Pleistoceno superior la Formación El Palmar (Zucol et al., 2005; Patterer, 2012; Patterer et al., 2014) en la Cuenca del río Uruguay, siendo el afloramiento de esta última formación geológica el que se relaciona con la actual presencia de los mayores núcleos de $B$. yatay, presentes como comunidades edáficas.

En la actualidad, los suelos arenosos pardos tales como la serie de suelos Puerto Yeruá desarrollados sobre antiguas terrazas del río Uruguay, poseen escasa presencia de comunidades de palmares y un mayor desarrollo de vegetación arbustiva-herbácea. El principal objetivo de este trabajo es conocer si mediante la composición fitolítica de suelos regionales de mayor desarrollo, se evidencian cambios fisonómicos del paisaje en etapas tempranas de sus ciclos pedológicos, y así determinar si estos resultados permitirán establecer su relación con la retracción de los palmares de yatay.

\section{MARCO GEOMORFOLÓGICO}

Los mayores núcleos de $B$. yatay se encuentran como comunidades de palmares densos en una fisonomía de sabana densa o bosque de palmares, ubicados en el paisaje sobre interfluvios secundarios y terrazas altas en donde afloran los niveles cuspidales de la Formación El Palmar (Iriondo, 1980). Dicha formación es un depósito fluvial del 
río Uruguay integrado por arenas de cauce que incluyen grandes lentes de gravas y cantos rodados; además de la facies de cauce, el sistema incluye depósitos en facies arenosas de inundación y de albardón configurando un complejo fluvial de Ilanura. En Entre Ríos, la unidad es una antigua Ilanura aluvial del río Uruguay en la que se distingue el paleocauce principal meandriforme, con 5-6 km de radio de curvatura y con ancho similar al cauce actual. En el presente, este depósito constituye la terraza alta del río Uruguay, muy disectada y bien definida a lo largo del curso inferior de los afluentes (Iriondo y Kröhling, 2008).

Desde el punto de vista fisiográfico, los palmares de yatay se encuentran en las terrazas antiguas erosionadas del río Uruguay, con afloramientos de cantos rodados (Tasi, 2009). Estas terrazas presentan suelos arenosos rojizos y suelos arenosos pardos sobre sedimentos aluviales antiguos, más arcillosos, con características muy variables a corta distancia. Los mismos se disponen en una franja irregular paralela a la costa del río, en un ancho que varía aproximadamente de 2 a $30 \mathrm{~km}$. Los sedimentos arcillosos lacustres están mezclados y redepositados con las arenas (en parte rojas, en parte amarillentas y también sectores más decolorados). Sobre los sedimentos lacustres el río depositó luego una capa de material arenoso de poco espesor. Los suelos de esta franja generalmente presentan horizontes superficiales arenosos a muy arenosos, pardos a pardo oscuros, que yacen sobre materiales densos muy poco permeables y penetrables, presentes entre 30 a $70 \mathrm{~cm}$ de profundidad. Localmente reciben el nombre de "suelos mestizos" (Tasi, 2009).

\section{MATERIALES Y MÉTODOS Área de estudio}

La secuencia pedológica estudiada se encuentra en cercanías del PNEP, en la cantera La Chola II, Ruta 14, Departamento Colón, Entre Ríos (32 $2^{\circ} 00^{\prime} 11,2^{\prime \prime}$ S; $58^{\circ} 11^{\prime}$ 26,1" 0) (Fig. 1). El clima de esta región es templado húmedo de llanura y se caracteriza por su condición de planicie abierta sin restricciones a la influencia de los vientos húmedos del noreste y el accionar de los vientos secos y refrigerantes del suroeste (causantes de los cambios repentinos en el estado del tiempo), y a los vientos del sureste (aire frío saturado de humedad, que da lugar a semanas enteras de cielo cubierto,
Iluvias y temperaturas muy estables). Este clima, caracterizado por su suavidad y ausencia de situaciones extremas, es el de mayor aptitud para el cultivo de cereales, forrajeras y la cría de ganado (Tasi, 2009). Dispone de una vegetación propia de la "Provincia fitogeográfica del EspinalDistritos del Ñandubay y del Tala" teniendo en común la gran mayoría de las especies arbóreas autóctonas como: Prosopis algarrobilla Griseb., Prosopis nigra Hieron., Acacia caven (Molina) Molina, Schinus longifolia (Lindl.) Speg., Celtis spinosa Spreng., Aspidosperma quebracho-blanco Schltr., Jodina rhombifolia (Hook. y Arn.) Reissek, etc. (Cabrera, 1973).

El perfil La Chola presenta una potencia aflorante de 1,70 m (Fig. 2) y responde en sus características a la clase de suelos arenosos pardos localmente llamados "mestizos"; posee horizontes superficiales muy arenosos, pardos a pardos oscuros que yacen sobre materiales densos, muy poco permeables y penetrables. Estos materiales provienen de sedimentos lacustres, arcillosos, grisáceos, mezclados y redepositados con material arenoso. Sus propiedades dependen principalmente del espesor y textura de la capa arenosa superficial y del tipo de material arcilloso subsuperficial. Una característica típica de los mismos es la amplia gama de variabilidad de rasgos que surgen como resultado de las diferencias locales en el régimen de sedimentación, las que posteriormente influyeron en los procesos pedogenéticos ulteriores. Estas diferencias se manifiestan a corta distancia, conformando muchas veces un "mosaico" de suelos. Los suelos arenosos pardos se encuentran principalmente cerca del río Uruguay, a continuación de la franja de suelos arenosos rojizos (Tasi, 2009).

\section{Muestreo y extracción de fitolitos}

De acuerdo con estas premisas se seleccionó un perfil representativo de estos Entisoles, que se describió en campo siguiendo las normas establecidas por USDA (Soil Survey Manual, Etchevehere, 1976). El muestreo del perfil La Chola se realizó en forma continua de piso a techo, tomando muestras equidistantes cada $10 \mathrm{~cm}$, con un volumen aproximado de 200 gr y un total de 10 muestras. Las muestras pedológicas fueron ingresadas al repositorio de muestras sedimentarias bajo la denominación CIDPALBO-MS del laboratorio de paleobotánica del CICYTTP-CONICET (Diamante, Entre Ríos). Las muestras CIDPALB01845-1841 


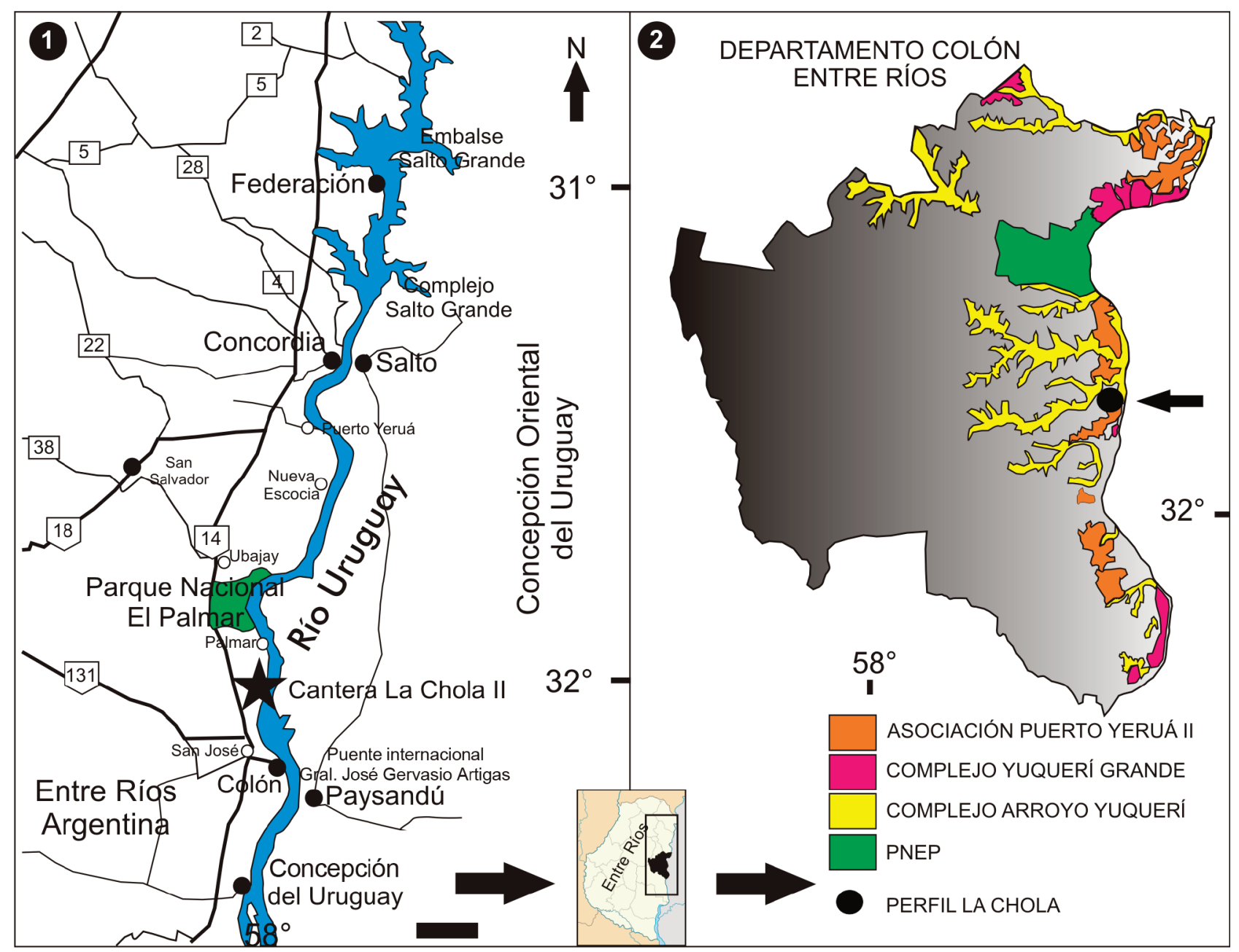

Figura 1. 1, Ubicación geográfica de la localidad fosilífera Cantera La Chola II, Colón, Entre Ríos, Argentina. 2, Mapa de suelos del departamento Colón mostrando las series de suelos relacionadas con los suelos arenosos pardos y suelos arenosos rojizos, tomado del Plan Mapa de Suelos (2002).

corresponden al horizonte A; las muestras CIDPALB018401837 al horizonte $B_{2}$ y la muestra CIDPALB01836 al horizonte C. En laboratorio las muestras fueron sometidas a procesamiento físico y químico con la finalidad de concentrar sus microrestos. Primero fueron secadas en estufa, luego se obtuvieron dos submuestras, una para archivo y otra que se trituró para ser sometida al tratamiento preliminar de acuerdo con Zucol et al. (2010). Los tratamientos preliminares tienen por finalidad la eliminación de toda sustancia que provoque la aglomeración de los elementos micropaleontológicos de manera de liberarlos para facilitar su extracción. Estos tratamientos consistieron en la eliminación de sales solubles mediante lavado con agua destilada, carbonatos con ácido clorhídrico 1 N, materia orgánica con agua oxigenada al 30\% y barnices y/o cementos con ácido clorhídrico $1 \mathrm{~N}$ en caliente. Después de una neutralización final del material remanente o "muestra limpia" se procedió a la desagregación química con hexametafosfato de sodio y posteriormente mecánica mediante agitador. Sobre la muestra limpia se realizó una separación granulométrica en tres fracciones de tamaño de partículas sobre las que se centraron los estudios fitolíticos. Las fracciones gruesa (mayor a $250 \mu \mathrm{m}$ de diámetro) y media (entre 53$250 \mu \mathrm{m}$ de diámetro) se separaron por tamizado en húmedo, mientras que la fracción fina (entre 5-53 $\mu \mathrm{m}$ de diámetro) se separó por sifonado a partir de la suspensión de la muestra dispersa en agua destilada. Las fracciones media y fina se sometieron a una separación densimétrica 
con solución de politungstato de sodio (densidad de 2,345 $\left.\mathrm{g} / \mathrm{cm}^{3}\right)$, extrayendo de esta manera el material liviano (incluyendo los fitolitos) presente en cada fracción. Este material concentrado se montó para su observación microscópica, tanto en medio líquido para observación tridimensional de los cuerpos, como en preparados permanentes con Bálsamo de Canadá. Se contabilizaron 400 elementos/muestra y se obtuvieron valores de abundancia relativa, con los que se creó una matriz básica de datos (MBD) sobre la que se efectuaron los análisis numéricos. Los morfotipos fitolíticos unicelulares fueron definidos usando la clasificación propuesta de Patterer et al. (2011), los cuales han sido dis- puestos en grupos mayores para su graficación (ver archivo suplementario Tabla 1, donde además se especifica la afinidad botánica de cada morfotipo, basados en las propuestas de Twiss et al. (1969), Bertoldi de Pomar (1971), Mulholland (1989), Twiss (1992), Kondo et al. (1994), Fredlund y Tieszen (1994) y Zucol (1996) y siguiendo las normativas y descriptores que enumera el ICPN Working Group (ICPNWG; Madella et al., 2005). El procesamiento de la información y su graficación en diagramas fitolíticos se realizaron con el programa POLPAL, Numerical Análysis (Walanus y Nalepka, 2000; Nalepka y Walanus, 2003).
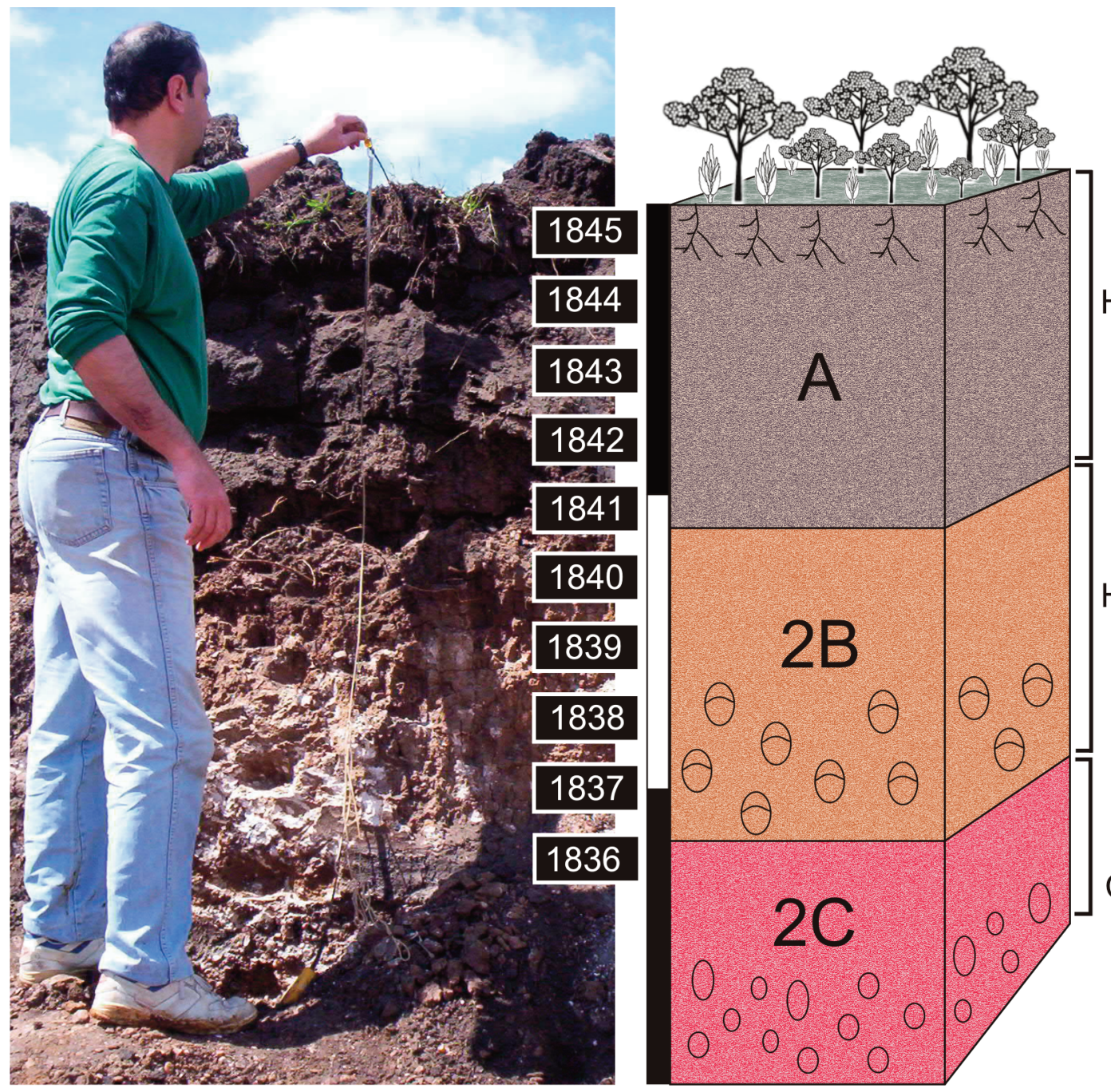

Horizonte A Orgánico

Horizonte B Textural $\mathrm{CaCO}_{3}$

Horizonte C Fm El Palmar Cantos rodados

Figura 2. Esquema del perfil pedológico La Chola y sus correspondientes ciclos pedológicos. Escala gráfica: $56,5 \mathrm{~cm}$. 


\section{RESULTADOS}

\section{Caracterización pedológica}

En el perfil estudiado (Fig. 2), el solum presenta una profundidad aproximada de 1,70 m, formado morfológicamente por un ciclo pedológico actual representado por un epipedón superficial profundo de aproximadamente 0,50 m, textura franco-arcillo-limosa, con presencia de abundante materia orgánica, grumosa, friable y una escasa presencia de cantos rodados en matriz. Los horizontes subsuperficiales de este tipo de suelos ("gredas") constan de más de un $80 \%$ de materiales lacustres arcillosos grisáceos, levemente mezclados con los arenosos. Son muy poco permeables y penetrables y muestran síntomas de hidromorfismo prácticamente en todo el perfil (Tasi, 2009).

Por debajo se desarrolla un segundo ciclo pedológico formado por un horizonte B textural, de textura francoarcillosa a arcillosa y estructuras prismáticas; densos con moteados y concreciones ferromanganesíferas y concreciones de carbonatos desde los 0,50-0,70 m. Este ciclo se asienta sobre sedimentos principalmente arenosos con una considerable presencia de cantos rodados los que estarían representando depósitos fluviales pleistocenos del río Uruguay (2C, Formación El Palmar).

Debido a las diferentes condiciones sedimentarias las características de los suelos arenosos pardos son tan variadas que, según el Sistema de la Taxonomía de Suelos (Soil Taxonomy), no es posible agruparlos en un solo taxa, ni como un nivel generalizado (Tasi, 2009).

\section{Caracterización fitolítica y afinidad botánica}

En lo referente a la variabilidad composicional de las asociaciones fitolíticas estimada mediante la rarefacción (Fig. 5) puede observarse que el perfil La Chola cuenta con dos grupos de asociaciones claramente diferenciadas, un grupo superior o PLC I (Perfil La Chola I) y uno inferior PLC II (Perfil La Chola II). En el punto de separación de los dos grupos, se encuentra la muestra 1841 que no resultó fértil para material silíceo y su composición textural era principalmente arcillosa.

PLC II: conjunto de asociaciones de las muestras 18361840, que representan niveles del segundo ciclo pedológico que se desarrollan a partir de los $70 \mathrm{~cm}$ del perfil hasta la base. Las muestras 1837-1840 fueron tomadas en el hori- zonte $2 \mathrm{~B}$ textural, mientras que la 1836 se asocia al horizonte $2 \mathrm{C}$, vinculada al material parental inicial de estos ciclos (principalmente relacionados con los depósitos de la Formación El Palmar). La asociación fitolítica quedó definida por la abundancia de fitolitos globulares de superficie espinosa cuyo diámetro promedio fue de $10 \mu \mathrm{m}$ (Fig. 4, 1-6), elementos que han sido descriptos en muestras de cenizas de hojas de especies vegetales de la familia Arecaceae, entre ellas Butia yatay (Patterer, 2014). Los fitolitos globulares espinosos presentan equivalencias clasificatorias tales como: "Echinate sphere" (Fenwick et al., 2011; Strömberg et al., 2013) y "Globular echinate" (Iriarte y Alonso Paz, 2009; Mercader et al., 2009; Benvenuto et al., 2015) y han sido descriptos no solo en muestras de vegetales actuales (Patterer, 2012, 2014), sino también en muestras sedimentarias que datan del Eoceno-Oligoceno y Mioceno (Zucol et al., 1999, 2001; Zucol y Brea, 2000a); Plio-Pleistoceno Temprano (Patterer, 2015); Pleistoceno Tardío (Zucol et al., 2005; Patterer, 2012; Patterer et al., 2014) y muestras de niveles pedológicos y horizontes orgánicos relacionadas directamente a unidades fisonómicas del PNEP (Patterer, 2012). Estos morfotipos con una media de $10 \mu \mathrm{m}$ de diámetro para Butia yatay (Patterer, 2014), pueden presentar diámetros que van desde los $2 \mu \mathrm{m}$ (en Jubaea chilensis (Molina) Baill, palma chilena) a $25 \mu \mathrm{m}$ (en Copernicia alba Morong), siendo la media general de diámetro no menor a $5 \mu \mathrm{m}$ (Patterer, 2014).

Acompañan la asociación, fitolitos en forma de conos truncados (principalmente $\mathrm{Ct03}, \mathrm{Ct0} 4$ y Ct05) de afinidad graminoide (con marcados componentes danthonioides); fitolitos graminoides no diagnósticos como los elongados de bordes lisos (Mp09, Fig. 3, 2-4) y ondulados (Mp08, Fig. 3 , 1), en forma de flabelos (FI, Fig. 3, 8 y 11), poliédricos (Sx, Fig. 3,5 y 10) y aguzados (Ac). En menor abundancia se observaron fitolitos en forma de silla de montar (Sm01) y halteriformes ( $\mathrm{Ha}$ ), que en ambos casos poseen afinidad a gramíneas megatérmicas (chloridoides y panicoides respectivamente). Es importante también considerar que en estas asociaciones se observaron morfotipos afines a especies dicotiledóneas, vinculados a vegetales arborescentes como fitolitos globulares a sub-globulares con superficie lisa (GIO2, Fig. 4, 7) o suavemente rugosa (GI05, Fig. 4, 8) de 10 ㅆm de diámetro promedio (Piperno, 1988; Runge, 1999; 
Collura y Neumman, 2016) y elementos de conducción. Los fitolitos de afinidad ciperoides (tales como Pi01, Pi02, Mp01 y Mp06) fueron escasos.
PLC I: el conjunto de asociaciones superiores (muestras 1842-1845) presentan una mayor variabilidad composicional, con una marcada abundancia de fitolitos graminoides

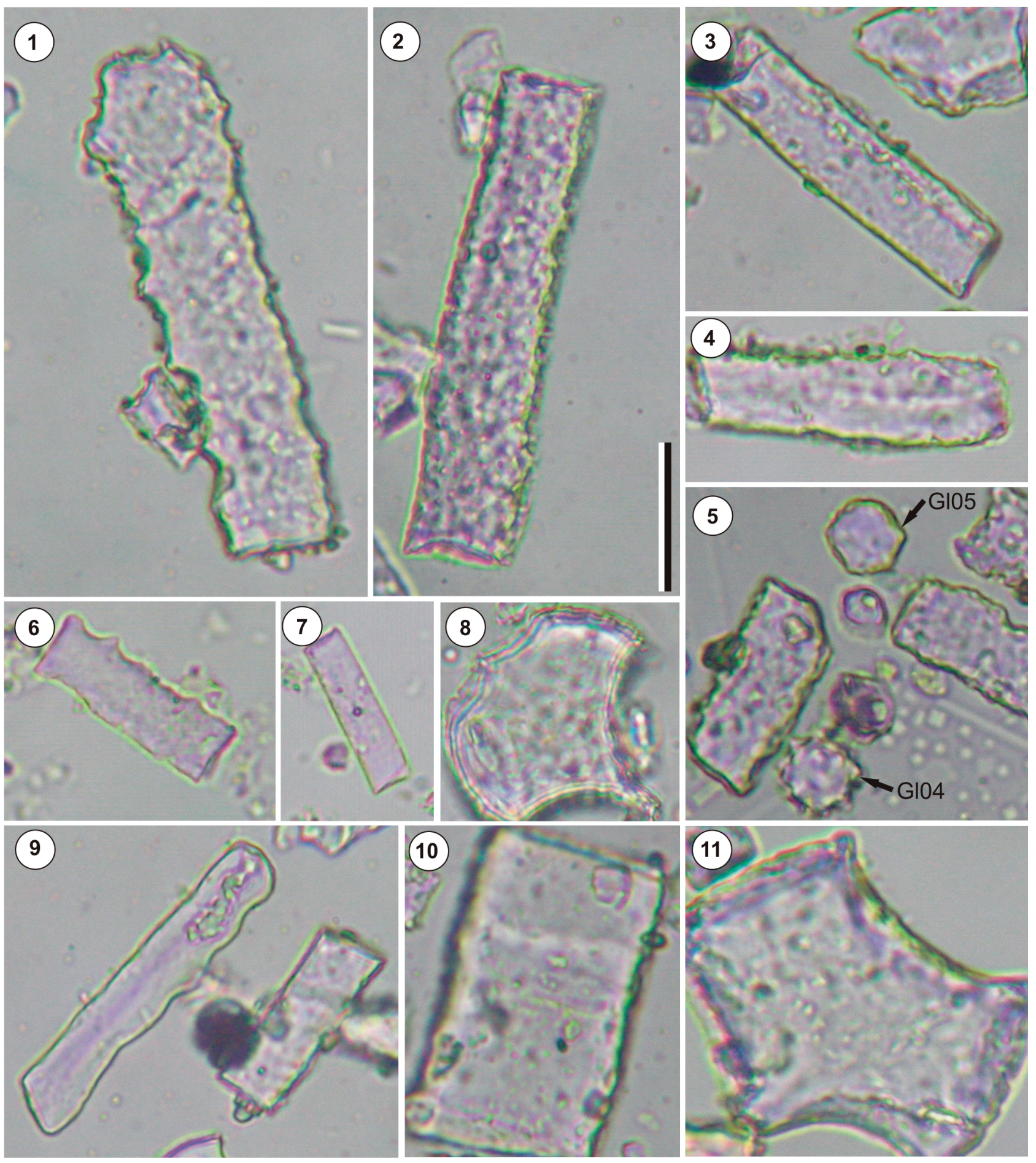

Figura 3. Lámina de fitolitos. 1, elongado largo de borde ondulado (Mp08); 2-4, elongado largo de borde liso (Mp09); 5, poliédrico chico (Mx02); 6, elongado corto de borde ondulado; 7, elongado corto de borde liso (MiO1); 8, flabelo (FI04); 9, en forma de bote largo; 10, poliédrico grande (SX02); 11, flabelo (FI06). Escala gráfica: $20 \mu \mathrm{m}$, válida para todas las fotos. 
(diagnósticos y no diagnósticos) y una considerable baja presencia de fitolitos globulares espinosos. Entre los fitolitos graminoides diagnósticos resultaron muy frecuente los tipos halteriformes (Fig. 4, 17-27) y en forma de cruz (principalmente los morfotipos Ch01, $\mathrm{HaO1}, \mathrm{HaO2}, \mathrm{HaO3}, \mathrm{HaO}$, Ha08, Ha09, Ha10, Ha11). Estos elementos son caracterís-
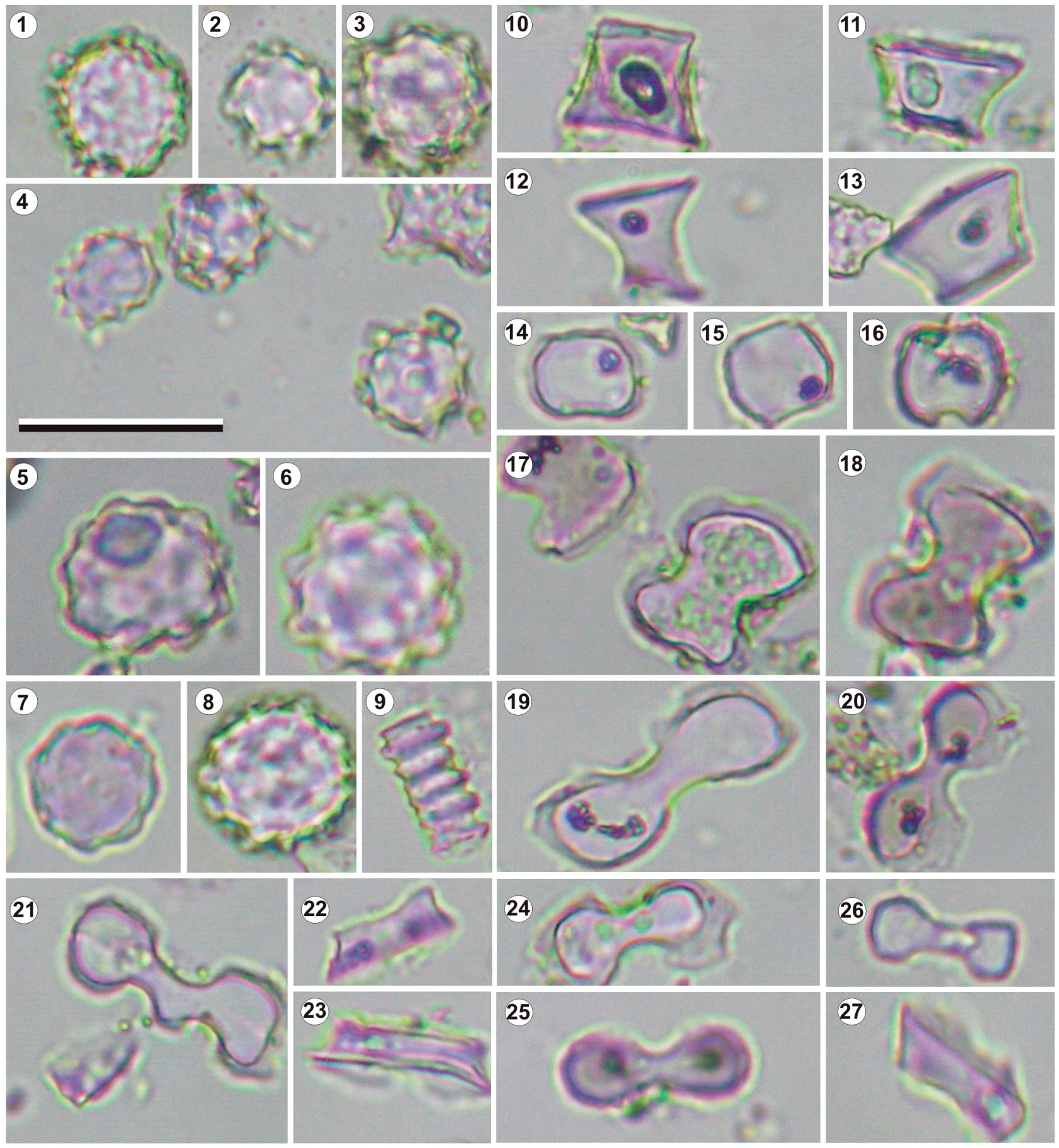

Figura 4. Lámina de fitolitos. 1-6, globulares espinosos (GI04); 7-8, globulares de superficie lisa o levemente rugosa (GI05); 9, elemento de conducción (Du02); 10, 11 y 13, conos truncados (CT03); 12, cono truncado (Ct05); 14-16, sillas de montar (Sm01); 17-18, halteriformes (Ha02), 19 y 20, halteriformes (Ha09); 21, halterio plurilobulado (PIO2); 22, 23 y 27, halteriformes en vista lateral; 24, halterio tipo-stipa; 25-26, halteriformes (Ha01). Escala gráfica: $20 \mu \mathrm{m}$, válida para todas las fotos. 
ticos de gramíneas panicoides y han sido citados en especies vegetales de los géneros Axonopus P. Beauv., Digitaria Haller, Paspalum L., Setaria P. Beauv., entre otras, y a suelos asociados a las unidades fisonómicas que conforman el área del PNEP (Patterer y Zucol, 2011; Patterer, 2012). Los fitolitos en forma de silla de montar fueron observados de forma frecuente, principalmente los morfotipos Sm01 y Sm02 (Fig. 4, 14-16), este tipo de elementos ha sido descripto en gramíneas chloridoides, tal es el caso de especies de los géneros Eragrostis Wolf. y Sporobolus R. Br. presentes en la unidad fisonómica pastizales del PNEP (Patterer, 2012). Halterios tipo-stipa (Mh01, Fig. 4, 24) y conos truncados (Ct03, Ct04, Ct07, Fig. 4, 10-13) fueron muy abundantes en esta sección.

Esta separación zonal del perfil queda expresada en el gráfico de asociación ConSLink (Fig. 5) que dividió al perfil en dos secciones claramente diferenciadas por las asociaciones fitolíticas que estarían caracterizando. Por un lado, una paleocomunidad de palmares y estrato herbáceo de gramíneas danthonoideas en los niveles inferiores del perfil, correspondientes al segundo ciclo pedológico, y por otro, una paleocomunidad de pastizales mega-mesotérmicos de gramíneas panicoideas, stipoideas, danthonoideas y en menor medida chloridoideas en los niveles superiores del ciclo pedológico reciente.

\section{CONSIDERACIONES FINALES}

El registro fósil de la Cuenca del río Uruguay evidencia la presencia de palmeras durante el Plio-Pleistoceno Temprano (Formación San Salvador), las cuales se encuentran asociadas a elementos arbóreos y gramíneas meso-megatérmicas junto a elementos bambusoides del sotobosque. El contexto del registro fósil permite definir asociaciones vegetales desarrolladas en las proximidades de cuerpos de aguas de rápidos o saltos, en microambientes húmedos con la presencia de plantas acuáticas y en un clima tropical con una importante disponibilidad hídrica (Patterer, 2015). Para el Pleistoceno Tardío (Formación El Palmar) el registro fósil permitió describir la presencia de palmares tanto de los tipos semidensos como densos, asociados a pastizales mega-mesotérmicos y elementos arbóreos que confirman la presencia de bosques mixtos húmedos y secos, que permiten inferir un clima tropical a subtropical húmedo (Zucol et al., 2005; Brea et al., 2010; Ramos et al., 2012; Patterer, 2012; Patterer et al., 2014). En el presente trabajo, el perfil La Chola se encuentra a 3,5 km del río Uruguay en la franja de suelos arenosos pardos (Serie Puerto Yeruá y Serie Mandisoví), contigua a la franja de suelos arenosos rojizos (Complejo Arroyo Yuquerí y Asociación Yuquerí Chico) y a aproximadamente $15 \mathrm{~km}$ del PNEP. La vegetación que actualmente se desarrolla en el área de estudio responde a la composición de los campos ganaderos vecinos del PNEP, con una fisonomía herbácea y sectores no aprovechados donde pueden evidenciarse restos de monte con presencia de arbustos y hasta árboles, fisonomía esta última que con la salvedad de la presencia de estípites resulta similar a la que se observa en las sabanas del área protegida, donde se desarrolla un conspicuo estrato arbustivo. Las palmeras en diferentes densidades (densos y semidensos) ocupan el estrato superior de la formación vegetal en la reservas de la región (Rolhauser et al., 2007; Lunazzi, 2009; Biganzoli, 2011; Chaneton et al., 2012; Rolhauser y Batista, 2014; Batista et al., 2014).

En ambas formaciones vegetales la relación herbáceas/palmeras varía, ello puede estimarse en las asociaciones fitolíticas, así la relación de abundancia de ambos conjuntos de tipos de fitolitos (gramíneas diagnósticas/no diagnósticas vs. arecoides) resulta de 29,22 $(37,53)$ 42,82\% de morfotipos pooides frente a $44,25(47,10) 51,64 \%$ de morfotipos arecoides en la sección inferior (PLC II), mientras que en la sección superior (PLC I) es de 76,36 $(81,67)$ 91,17\% de morfotipos pooides frente a $2,10(2,92) 4,18 \%$ de morfotipos arecoides. Esta elevada relación o presencia de fitolitos de palmeras ha sido observada en muestras superficiales de suelo de las unidades fisonómicas palmares semidensos y densos en el área del PNEP con valores de abundancia porcentual de morfotipos fitolíticos arecoideos entre un 50 a $70 \%$ y un 40 a $50 \%$ de morfotipos pooides (Patterer, 2012). Este tipo de comunidad presenta, en su mayoría, fisonomía de sabana densa o de bosque de palmeras y generalmente se localiza en terrazas altas e interfluvios secundarios. El estrato arbóreo superior cubre en promedio el $25 \%$ de la superficie y está conformado por individuos adultos de Butia yatay y ocasionalmente algún árbol de otra especie nativa. En el estrato intermedio (cobertura promedio $32 \%$ ) dominan especies arbustivas como 
Baccharis dracunculifolia DC. y B. pingraea DC., a los que frecuentemente se agregan especies arbóreas como Eugenia myrcianthes Nied., Allophylus edulis (A.St.-Hil., A.Juss. y Cambess.) Radlk., Guettarda uruguensis Cham. y Schltdl. y Maytenus ilicifolia Mart. ex Reissek e individuos altos del pasto nativo Andropogon lateralis Nees. El estrato herbáceo es relativamente continuo (cobertura promedio $73 \%$ ) y aparece dominado por el helecho Adiantopsis chlorophylla (Sw.) Fée y el pasto cespitoso Bromus auleticus Trin. ex Nees (Batista et al., 2014). Según los registros de la Administración de Parques Nacionales (APN), la mayor parte de los sitios de esta comunidad habían sido afectados por fuego por última vez a fines de la década de 1970 o en la década de 1990. Por su parte, los porcentajes de fitolitos con afinidad a dicotiledóneas arbóreas resultaron de $0(2,85) 5,04 \%$ en la PLC II y $0(0,41) 1,04 \%$ en la PLC I, lo cual demuestra una mayor abundancia de elementos arbóreos conjuntamente a los arecoides en la sección inferior.
Luego del sector transicional que no ha presentado microrestos, se observa un demarcado cambio composicional en el ciclo superior (PLC I) de reciente formación, que estaría en relación a una paleocomunidad caracterizada por una importante abundancia de elementos graminoides y escasa a nula presencia de elementos arbóreos y arecoides. Los tipos morfológicos observados con cambios en composición fitolítica puntual de algunos morfotipos, se asimilan a los encontrados en secuencias superficiales de la unidad fisonómica pastizales en el PNEP, con abundancias porcentuales de morfotipos pooides del $50 \%$ vs. un $20 \%$ de fitolitos arecoideos. Estas comunidades denominadas "estepas psamófilas" (Batista et al., 2014) presentan fisonomía de pastizal o estepa graminosa, con algunas palmeras altas muy aisladas. El estrato arbóreo inferior está siempre ausente. El intermedio, habitualmente ausente, aparece integrado muy ocasionalmente por escasos individuos de Baccharis dracunculifolia, Pavonia hastata Cav. o pastos como Bromus

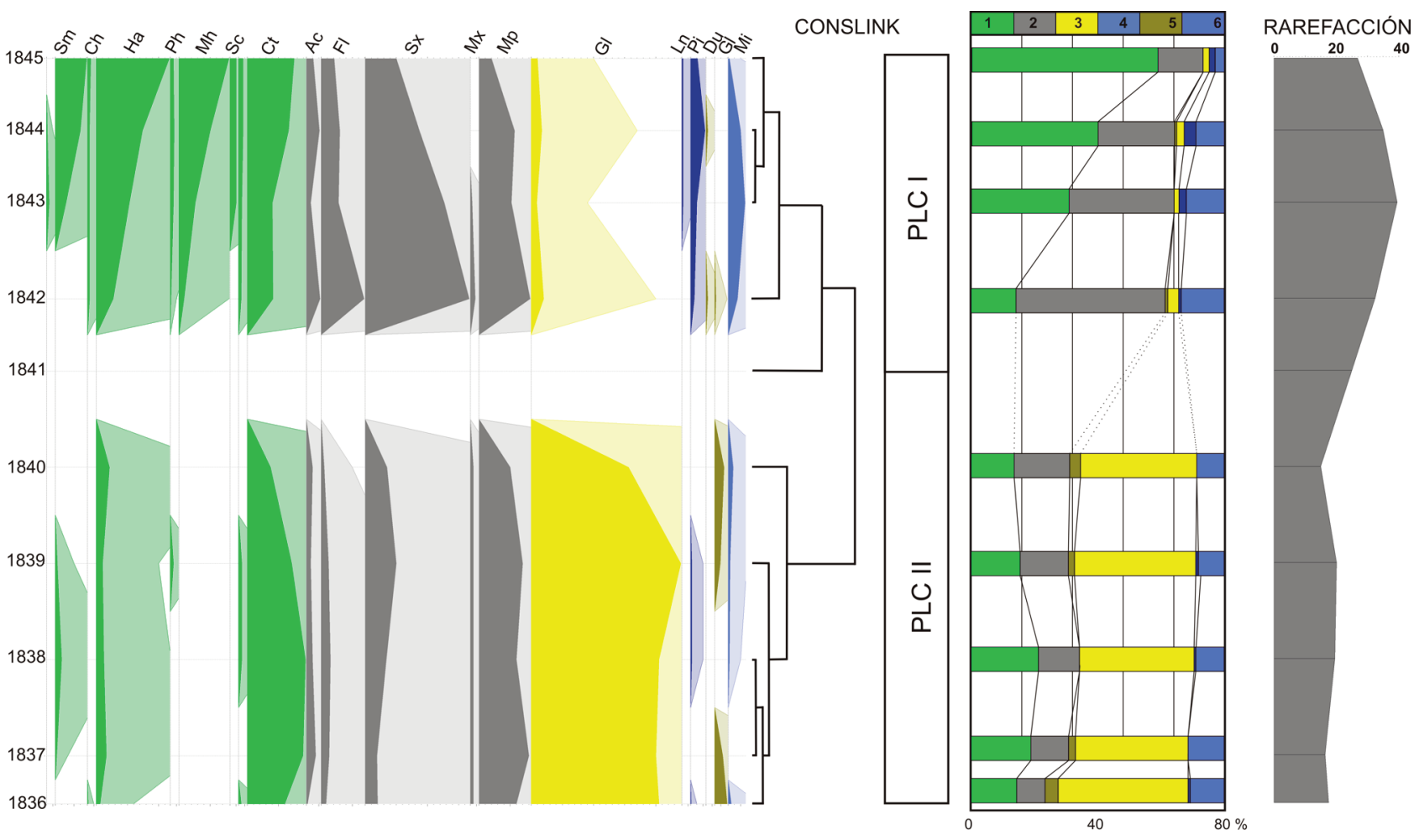

Figura 5. Diagrama fitolítico con los principales morfotipos fitolíticos, CosLink o gráfico de asociación zonificando el perfil en PLC I y PLC II y rarefacción mostrando la diversidad de morfotipos presente por muestra. Referencias: 1. Gramíneas diagnósticas; 2. Gramíneas no diagnósticas; 3. Palmeras; 4. Dicotiledóneas arbóreas; 5. Herbáceas húmedas; 6. No diagnósticas; PLC I, Perfil La Chola I; PLC II, Perfil La Chola II. 
auleticus o Melica brasiliana Ard. El estrato herbáceo es muy característico, alcanza a cubrir en promedio el 83\% de la superficie y está dominado por los pastos Schizachyrium microstachyum (Desv. ex Ham.) Roseng., B.R. Arrill. \& Izag., Bromus auleticus, las hierbas Monnina cuneata A. St.-Hil., Plantago brasiliensis Sims. y el subarbusto Croton laureltyanus Ahumada. Es relativamente frecuente encontrar plántulas de Butia yatay. Esta comunidad aparece asociada con los profundos depósitos de arena masiva de la Formación El Palmar, que se ubican alrededor de las cabeceras de cañadas afluentes de los arroyos (Batista et al., 2014). Estos pastizales se encuentran topográficamente en sectores del paisaje con condiciones más benignas, ya sea por una mayor exposición per se o por condiciones del paisaje, lo que favorece la proliferación de gramíneas megatérmicas húmedas o más xéricas de acuerdo a la disponibilidad hídrica.

\section{CONCLUSIÓN}

Tanto la información paleobotánica como los registros históricos y de los primeros naturalistas, permiten evidenciar una mayor presencia y distribución de las palmeras, en particular los palmares de Butia yatay. En el lapso histórico, parte de esta retracción se vincula con el aprovechamiento directo de estos vegetales, como así también la actividad humana que indirectamente ha impedido su conservación o desarrollo, sin considerar en ello posibles cambios climáticos en mayor escala temporal que podrían estar favoreciendo esta retracción. Los resultados obtenidos mediante el registro fitolítico del perfil La Chola demarcó para una misma área el cambio en la fisonomía del paisaje, pasando de una comunidad de palmares a una más reciente de pastizales.

Los resultados obtenidos han permitido no solo caracterizar la secuencia pedológica de esta región, la que a priori resultaba de difícil interpretación en campo, sino también reconocer que las variaciones pedológicas se corroboran con un cambio de la vegetación que se desarrollaba en sus ciclos. La presencia de asociaciones tan disímiles muestra una clara presencia de dos ciclos edáficos separados probablemente por una discontinuidad en el desarrollo del suelo, provocada por algún tipo de evento erosivo importante que terminó por enterrar parte del suelo sobre el cual comenzó a depositarse el epipedón actual. Serían necesarios datos de edades absolutas y el muestreo de nuevos perfiles en suelos arenosos pardos, ampliando el área de estudio y de prospección de suelos arenosos rojizos para tratar de reconstruir la historia de los palmares en la provincia de Entre Ríos a nivel regional. Estos antecedentes presentan una valiosa herramienta para el conocimiento de la historia evolutiva reciente de los palmares en la región litoral argentina.

\section{BIBLIOGRAFÍA}

Báez, J.R. 1937. Área de dispersión actual de las Palmáceas en la flora de Entre Ríos. Anales de la Sociedad Argentina de Estudios Geográficos 5: 63-78.

Báez, J.R. 1942. Las palmeras de la flora de Entre Ríos. Museo de Historia Natural de Entre Ríos. Ministerio de Agricultura de la Nación, Talleres Gráficos, Casa Radio-Paraná Museo de Entre Ríos, $10 \mathrm{p}$.

Batista, W.B., Rolhauser, A.G., Biganzoli, F., Burkart, S.E., Goveto, L., Maranta, A., Pignataro, A.G., Morandeira, N.S. y Rabadán, M. 2014. Las comunidades vegetales de la sabana del Parque Nacional El Palmar (Argentina). Darwiniana, nueva serie 2: 5-38.

Benvenuto, M.L., Fernández Honaine, M., Osterrieth, M.L. y Morel, E. 2015. Differentiation of globular phytoliths in Arecaceae and other monocotyledons: morphological description for paleobotanical application. Turkish Journal of Botany 39: 341-353. Doi:10.3906/bot-1312-72

Bertoldi de Pomar, H. 1971. Ensayo de clasificación morfológica de los silicofitolitos. Ameghiniana 8: 317-328.

Biganzoli, F. 2011. [Influencia de los incendios en la dinámica poblacional de dos arbustos dominantes en la sabana mesopotámica. Tesis Doctoral, Facultad de Agronomía, Universidad de Buenos Aires, Buenos Aires, 151 p. Inédita.].

Brea, M., Zucol, A.F. y Patterer, N.I. 2010. Fossil woods from late Pleistocene sediments from El Palmar Formation, Uruguay Basin, Eastern Argentina. Review of Palaeobotany and Palynology 163: 35-91.

Cabrera, A.L. 1973. Esquema fitogeográfico de la República Argentina. Revista del Museo de La Plata (Nueva Serie), Botánica, 8: 87-168.

Chaneton, E.J., Mazia, N., Batista, W.B., Rolhauser, A.G. y Ghersa, C.M. 2012. Woody plant invasions in Pampa grasslands: a biogeographical and community assembly perspective. En: R.W. Myster (Ed.), Ecotones between Forest and Grassland. Springer, New York, p. 115-144.

Collura, L.V. y Neumman, K. 2016. Wood and bark phytoliths of West African woody plants. Quaternary International 434: $142-159$.

Crovetto, R. y Piccinni, B. 1951. La vegetación de la República Argentina. I. Los Palmares de Butia yatay. Revista de Investigación Agrícola 4: 153-242.

Eguía, G., Paparotti, O., Tasi, H. y Bresseur, N. 2002. Plan Mapa de Suelos. Carta de suelos de la República Argentina. Departamento Colón. Ediciones INTA. CD-ROOM.

Erra, G. 2010. Asignación sistemática y paleocomunidades inferidas a partir del estudio fitolítico de sedimentos cuaternarios de Entre Ríos, Argentina. Boletín de la Sociedad Argentina de Botánica 45: 309-319.

Erra, G., Zucol, A.F. y Kröhling, D. 2011. Análisis fitolítico de la Formación Tezanos Pinto (Pleistoceno tardío- Holoceno temprano) 
en el sector noroeste de su área de distribución en la provincia de Entre Ríos (Argentina). Revista Mexicana de Ciencias Geológicas 28: 398-412.

Erra, G., Zucol, A.F., Kröhling, D. y González, C.A. 2013. Análisis Fitolítico de la Formación Tezanos Pinto (Pleistoceno tardíoHoloceno temprano) en el sector sudoeste de la provincia de Entre Ríos (Argentina). Ameghiniana 50: 429-446.

Etchevehere, P. 1976. Normas de reconocimiento de suelos. Instituto Nacional de Tecnología Agropecuaria, Departamento de Suelos, Ciudad Autónoma de Buenos Aires, 152: 211 p.

Fenwick, R.S.H., Lentfer, C.J. y Weisler, I.M. 2011. Palm reading: A pilot study to discriminate phytoliths of four Arecaceae (Palmae) taxa. Journal Archaeological Science 38: 2190-2199.

Fredlund, G.G. y Tieszen, L.T. 1994. Modern phytolith assemblages from the North American Great Plains. Journal Biogeography 21: 321-335.

Goveto, L. 2005. [Ocurrencia histórica de fuegos en la sabana del Parque Nacional El Palmar: evidencias climáticas y florísticas. Tesis de maestría, Facultad de Agronomía, Universidad de Buenos Aires, Buenos Aires, 115 p. Inédita.].

Iriarte, J. y Alonso Paz, E. 2009. Phytolith analysis of selected native plants and modern soils from southeastern Uruguay and its implications for paleoenvironmental and archeological reconstruction. Quaternary International 193: 99-123.

Iriondo, M.H. 1980. El cuaternario de Entre Ríos. Revista de la Asociación de Ciencias Naturales del Litoral 11: 125-141.

Iriondo, M.H. y Kröhling, D. 2008. Cambios ambientales en la cuenca del Uruguay (desde el Presente hasta dos millones de años atrás). Colección Ciencia y Técnica, Universidad Nacional del Litoral, Santa Fe, $330 \mathrm{p}$.

Kondo, R., Child, C., y Atkinson, I. 1994. Opal phytoliths of New Zealand. Maanaki Whenua Press, Lincoln, 85 p.

Lunazzi, M.M. 2009. [Estructura y dinámica poblacional de la palmera Butia yatay en la sabana del Parque Nacional El Palmar: análisis en la escala de stand. Tesis de Maestría, Facultad de Agronomía, Universidad de Buenos Aires, Buenos Aires, 129 p. Inédita.].

Madella, M., Allexandre, A.y Ball, T. 2005. International Code for Phytolith Nomenclature 526 1.0. Annals of Botany 96: 253-260.

Mercader, J., Bennett, T., Esselmont, C., Simpson, S. y Walde, D. 2009. Phytoliths in woody plants from the Miombo woodlands of Mozambique. Annals of Botany 104: 91-113.

Mulholland, S.C. 1989. Phytolith shape frequencies in North Dakota grasses: a comparison to general patterns. Journal Archaeology Science 16: 489-511.

Nalepka, D. y Walanus. A. 2003. Data processing in pollen analysis. Acta Paleobotánica 43: 125-134.

Patterer, N.I. 2012. [Análisis Fitolíticos de la Formación El Palmar (Pleistoceno superior) en la cuenca del río Uruguay (este de Argentina). Tesis Doctoral, Universidad Nacional de Córdoba, Córdoba, 307 p. Inédita.].

Patterer, N.I. 2014. Análisis fitolíticos de las principales especies de palmeras (Arecaceae) presentes en regiones subtropicales de América del Sur. Boletín de la Sociedad Argentina de Botánica 49: 491-502.

Patterer, N.I. 2015. Análisis fitolíticos de la Formación San Salvador (Plioceno-Pleistoceno inferior) en la Cuenca del río Uruguay, Entre Ríos, Argentina. Revista de la Asociación Geológica Argentina 72: 332-344.

Patterer, N.I. y Zucol, A.F. 2011. Plant Communities Phytolith Assemblages relationships with native flora from El Palmar National Park (Entre Ríos province, Argentina). $8^{\circ}$ International
Meeting on Phytolith Research (Estes Park, Colorado), Abstracts: 43-44.

Patterer, N.I., Passeggi, E. y Zucol, A.F. 2011. Análisis fitolíticos de suelos del sudoeste de la Provincia de Entre Ríos (Argentina) como una herramienta para comprender sus procesos pedológicos. Revista Mexicana de Ciencias Geológicas 28: 132-146.

Patterer, N.I., Zucol, A.F. y Brea, M. 2014. Análisis fitolíticos del perfil sedimentario Los Loros, de la Formación El Palmar (Pleistoceno superior), en el Parque Nacional El Palmar, Entre Ríos, Argentina. Acta Geológica Lilloana 26: 53-62.

Pearsall, D.M. 2000. Paleoethnobotany. A handbook of procedures. $2^{\circ}$ ed. Academic Press, New York, 700 p.

Piperno, D.R. 1988. Phytolith analysis: an archaeological and geological perspective. Academic Press, San Diego, $280 \mathrm{p}$.

Piperno, D.R. 1998. Phytoliths. En: J.P. Smol, H.J. Birks, W.M. Last (Eds.), Tracking Environmental Change Using Lake Sediments. Volume 3: Terrestrial, Algal, and Siliceous Indicators. Developments in Paleoenvironmental Research 3, Springer, Dordrecht, p. 235-251.

Ramos, R.S., Brea, M. y Kröhling, D.M. 2012. Leños fósiles de la Formación El Palmar (Pleistoceno Tardío) en el parque Nacional El Palmar, Entre Ríos, Argentina. Ameghiniana 49: 606-622.

Rolhauser, A.G. 2002. [Expansión de poblaciones arbóreas nativas en las sabanas del Parque Nacional El Palmar: patrones y procesos en las escalas de paisaje y de parche. Tesis de Maestría, Facultad de Agronomía, Universidad de Buenos Aires, Buenos Aires, 68 p. Inédita.].

Rolhauser, A.G. y Batista, W.B. 2014. From pattern to process: estimating expansion rates of a forest tree species in a protected palm savanna. Landscape Ecology 29: 919-931.

Rolhauser, A.G., Biganzoli, F., Pignataro, A.G., Nordenstahl, M. y Batista, W.B. 2007. Distribución de las poblaciones arbóreas en el paisaje del Parque Nacional El Palmar. En: S.D. Matteucci (Ed.), Panorama de la ecología de paisajes en Argentina y países sudamericanos, Ediciones INTA, Buenos Aires, p. 329-334.

Runge, F., 1999. The opal phytolith inventory of soils in central Africa -quantities, shapes, classification, and spectra. Review Palaeobotany and Palynolinology 107: 23-53.

Strömberg, C.A.E., Regan, E.D., Madden, H.R., Matthew, J.K., Kohn, J. y Carlini, A.A. 2013. Decoupling the spread of grasslands from the evolution of grazer-type herbivores in South America. $\mathrm{Na}$ ture communications 4: 1478. Doi: $10.1038 /$ ncomms 2508

Tasi, H.A. 2009. [Aplicación de las Cartas de Suelos de Entre Ríos, Argentina, para evaluar Índices de Productividad Específicos para los principales Cultivos Agrícolas. Tesis Doctoral, Universidade Da Coruña, Coruña, España. 618 p. Inédita.].

Tomlinson, P.B. 1961. Anatomy of Monocotyldons. Il: Palmae. Clarendon Press, Oxford, $453 \mathrm{p}$.

Twiss, P.C., 1992. Predicted world distribution of C3 and C4 grass phytoliths. En: G. Rapp, Jr. y S.C. Mulholland (Eds.), Phytoliths Systematics. Emerging Issues. Advances in Archaeological and Museum Science 1, Springer, New York, p. 113-128.

Twiss, P.C., Suess, E. y Smith, R.M. 1969, Morphological classification of grass phytoliths. Soil Science of America Proceeding 33: 109-115.

Walanus, A. y Nalepka, D. 2000. POLPAL. Counting pollen, tables storage and diagrams plotting. System manual. Polish Academy of Sciences W. Szafer Institute of Botany, Cracovia, p. 25.

Zucol, A.F. 1996. Microfitolitos de las Poaceae argentinas: I. Microfitolitos foliares de algunas especies del género Stipa (Stipeae: Arundinoideae), de la Provincia de Entre Ríos. Darwiniana 34: $151-172$. 
Zucol, A.F. y Brea, M. 2000a. Análisis fitolítico de la Formación Paraná (Mioceno superior) en el Departamento Diamante, Entre Ríos, Argentina. $2^{\circ}$ Congreso Sedimentológico Latinoamericano y $8^{\circ}$ Reunión Argentina de Sedimentología (Mar del Plata), Resúmenes, p. 190-191.

Zucol, A.F. y Brea, M. 2000b. Análisis fitolítico de la Formación Paraná en Entre Ríos. En: F.G. Aceñolaza y R. Herbst (Eds.), El Neógeno de Argentina. INSUGEO, Serie de Correlaciones Geológicas 14, Tucumán, p. 67-76.

Zucol, A.F. y Brea, M. 2001. Asociación fitolítica de la Formación Alvear (Pleistoceno Inferior), Entre Ríos, Argentina. Ameghiniana, Suplemento-Resúmenes 38: 49R.

Zucol, A.F., Mazzoni, M.M. y Madden, R.H. 1999. Análisis fitolíticos en la secuencia sedimentaria de Gran Barranca, Chubut. Ameghiniana, Suplemento-Resúmenes 36: 43R.

Zucol, A.F., Brea, M., Carlini, A.A. y Madden R.H. 2001. Análisis fitolíticos en la secuencia sedimentaria de Gran Barranca, Chubut, Argentina: II. El Miembro Colhué Huapi (Formación Sarmiento). Ameghiniana, Suplemento-Resúmenes 38: 50R.

Zucol, A.F., Brea, M. y Scopel, A. 2005. First record of fossil Wood and phytoliths assemblages of the Upper Pleistocene in El Palmar National Park (Argentina). Journal American Earth Sciences 20: $33-43$.
Zucol, A.F., Passeggi, E., Brea, M., Patterer, N.I, Fernández Pepi, M.G. y Colobig, M.M. 2010. Phytolith analysis for the Potrok Aike Lake Drilling Project: sample treatment protocols for the pasado microfossil manual". En: H. Corbella y N.I. Maidana, (Eds.), $1^{\circ}$ Reunión Internodos del Proyecto Interdisciplinario Patagonia Austral y $1^{\circ}$ Workshop Argentino del Proyecto Potrok Aike Mar Lake Sediment Archive Drilling Project. Proyecto Editorial PIPA (Buenos Aires), p. 81-84.

Doi: 10.5710/PEAPA.20.03.2017.122

Recibido: 16 de agosto de 2016

Aceptado: 20 de marzo de 2017 